\title{
Innate immune function of serine/threonine-protein kinase from Macrobrachium rosenbergii in response to host-pathogen interactions
}

\begin{abstract}
The occurrences of multiple drug-resistant strains have been relentlessly increasing in recent years. The aquaculture industry has encountered major disease outbreaks and crucially affected by this situation. The usage of non-specific chemicals and antibiotics expedites the stimulation of resistant strains. Triggering the natural defense mechanism would provide an effective and safest way of protecting the host system. Hence, we have investigated the innate immune function of serine/threonine-protein kinase (STPK) in Macrobrachium rosenbergii (Mr). The in-silico protein analysis resulted in the identification of cationic antimicrobial peptide, MrSL19 , with interesting properties from STPK of M. rosenbergii. Antimicrobial assay, FACS and SEM analysis demonstrated that the peptide potentially inhibits Staphylococcus aureus by interacting with its membrane. The toxic study on MrSL-19 demonstrated that the peptide is not toxic against HEK293 cells as well as human erythrocytes. This investigation showed the significant innate immune property of an efficient cationic antimicrobial peptide, MrSL-19 of STPK from M. rosenbergii.
\end{abstract}

Keyword: Serine threonine-protein kinase; Macrobrachium rosenbergii; Cationic antimicrobial peptide; Membrane disruption; Innate immunity 